\title{
The E.E.M. in the recovery of muscle injuries
}

Keywords: EEM, Muscular electrostimulation; USG, Ultrasonographic

\section{The purpose of the study}

The objective of the study is to demonstrate how the Muscular Electrostimulation provides an important help in the recovery of muscular injuries starting from the Acute Stage. Athletes who presented a grade I and II muscle injury were subjected to E.E.M in the early stages of their recovery (acute).

Check under USG control of the degree of mobilization of muscle fibers in relation to the intensities and programs used. Clinical evaluation after applying the EEM and appreciation by the patient.

\section{Introduction}

Referential theoretical framework

A. Organization chart of the mechanism of muscle injury.

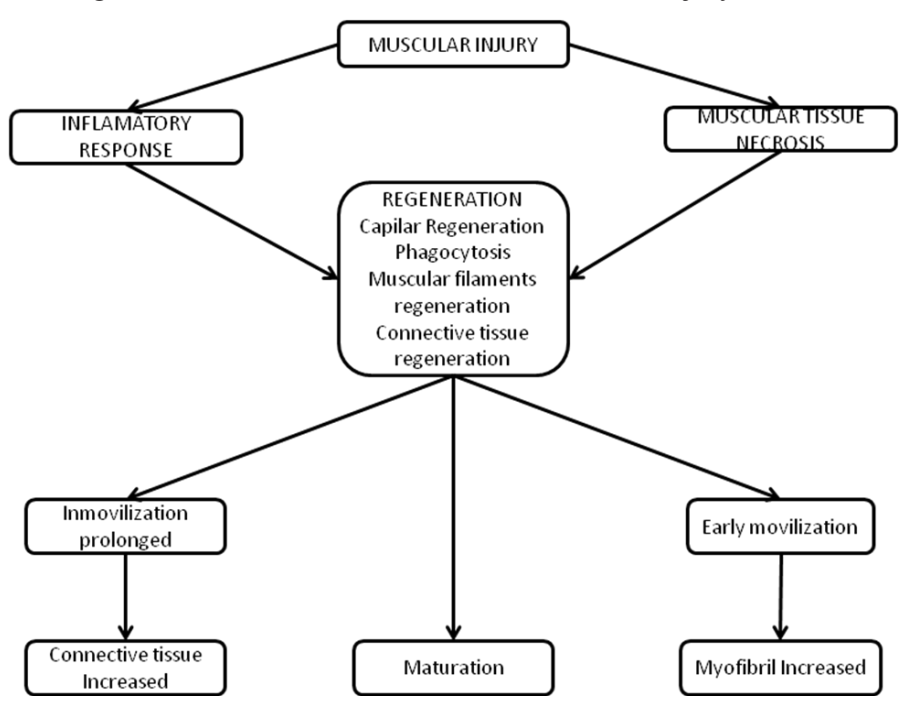

B. Repair vs. regeneration

I. Repair (ESPONTANEA): It is the restoration of this tissue without it conserving its original architecture or its function, the final result is healing.

II. Regeneration (induced): Limited capacity of certain tissues, when their restoration has properties indistinguishable from the original tissue.

i. We are interested as physiotherapists to promote regeneration over reparation.

ii. The circumstances under which a tissue heals instead of regenerating will depend on the content of cells and stimulating signals necessary for regeneration. ${ }^{1}$

iii. Under the membrane or sarcolemma are cell nuclei, about 40 per millimeter of cell, some represent satellite cells or INACTIVOS myoblasts that may have an important role in cell regeneration.
Volume 10 Issue $6-2018$

Carlos Alberto Coccaro Pinazo 1,2,3,4

'Bachelor of Physiotherapy, University of the Republic, Uruguay

${ }^{2}$ Specialist in Orthopedic Medicine (James Ciriax), Mexico

${ }^{3}$ Physiotherapist National Team of Mexico, Physiotherapist

Club de Fútbol Pachuca, Member of the Association of

Fisioterapeutas Del Uruguay, Uruguay

${ }^{4}$ Representative partner in Mexico of the Association of

Kinesiology of Sports of Argentina, Mexico

Correspondence: Carlos Alberto Cóccaro Pinazo, University of Soccer, Circuit of the Conception km2 s/n, Col. La Concepción, C.P. 42160, San Agustín Tlaxiaca, Hidalgo, Mexico, Email carloscocaro@hotmail.com

Received: January 31, 2018| Published: November 16, 2018

C. Possible roles of satellite cells in skeletal muscle adaptation (KADIETAL, 1999).

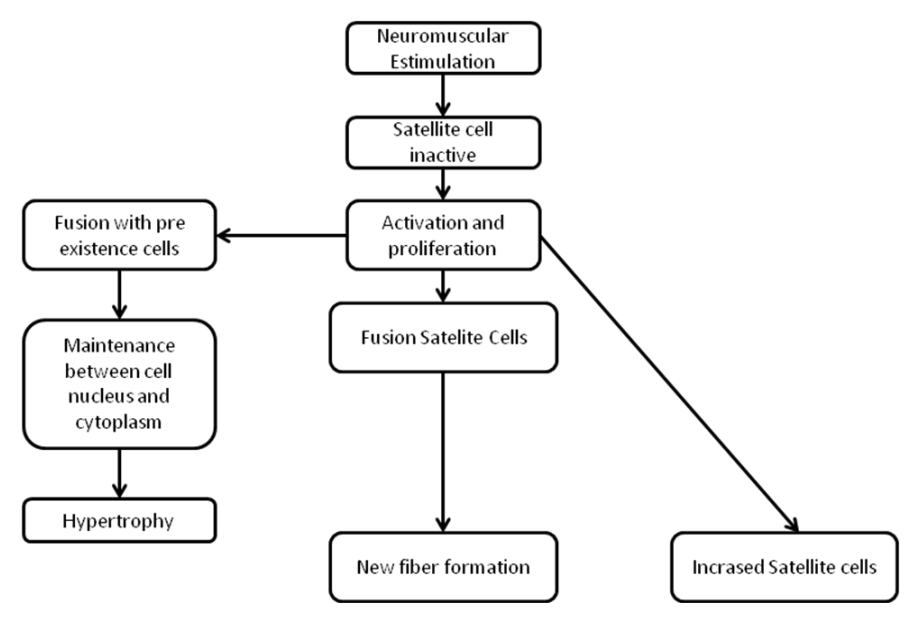

D. Regeneration of muscle tissue

a) Skeletal muscle: It regenerates from the satellite cells, which lie within the basal lamina that surrounds the muscle. Faced with muscle damage or other stimuli, these cells are activated, this ability varies from person to person.

b) Cardiac muscle: Hhas very little (almost nothing) ability to regenerate, is repaired by proliferation of connective tissue (scar).

c) Smooth muscle: Cápac. Moderate, if the proliferation is not enough to repair the damage occurs (scar).

That is, the cell loss itself stimulates proliferation by a homeostatic mechanism. The new capillaries are formed by budding and the growth is controlled by the factors released by the surrounding tissues. The bone marrow is the source of precursor cells with the ability to differentiate into different types of cells. These are called multipotentials, such as the following: osteoblasts, chondroblasts, myoblasts, etc. 


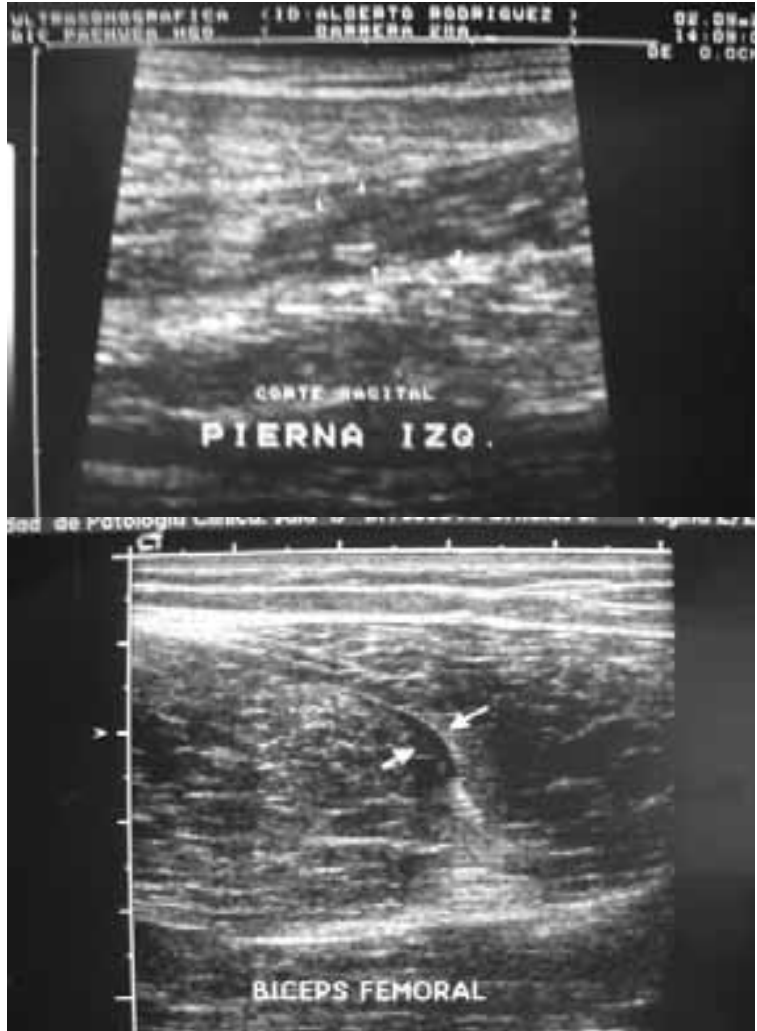

Figure 1\&2 Example of sight muscle injury in ultrasound.

\section{E. Effects of early mobilization}

a. One of the most effective methods for the regeneration of breaks and muscular contractions is to move the damaged part during the first stages of healing, because it generates very few complications and incapacities when compared with other immobilization treatments. ${ }^{2}$

b. The ultrastructural alterations that take place after the immobilization of the muscles, include a segmental necrosis that affects the extremities of the muscular fiber, ${ }^{3}$ being the type I fibers those that will suffer the most intense transformations. ${ }^{4}$

c. A finding that usually accompanies immobilization is the increase in connective tissue (endomysium, perimysium, epimysium) in relation to muscle contractile tissues.

d. This connective tissue does not align parallel to the muscle fibers, so that this increase in its size and structure will increase the muscle's rigidity, decreasing its extensibility properties. ${ }^{5}$

e. Traction and stretching in a slow way, stimulate the formation of new contractile tissue with an increase in the number of sarcomeres and the physiological cross-sectional area.

f. Causing little fibrosis and stiffness around the muscle fiber. ${ }^{6}$

g. Low-speed eccentric training increases the number of sarcomere formation in series and minimizes the proliferation of collagen, it is also a great stimulator of free nerve endings.

h. When the mobilization begins after a brief period of immobilization, it is observed that the penetration of the muscle fibers in the connective tissue is greater. ${ }^{7}$

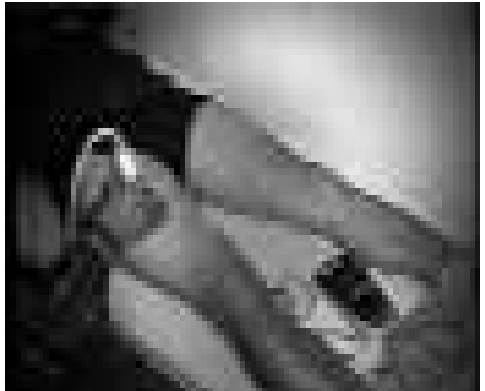

Figure 3 EEM verifying stimuli and mobilization of muscle fibers with ultrasound.

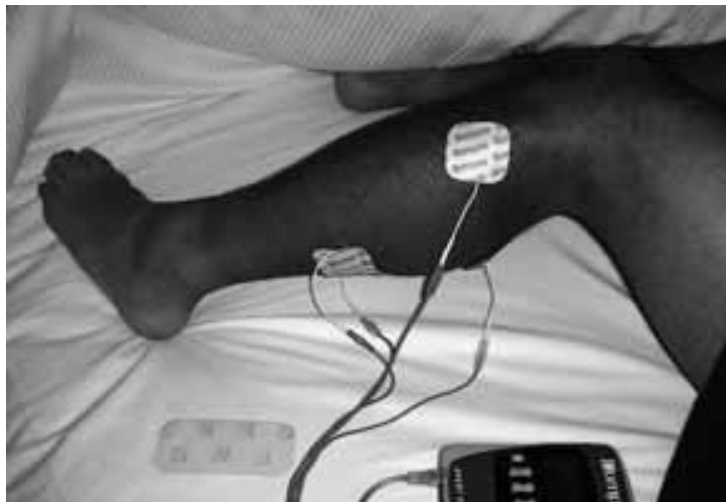

Figure 4 Typical example blow in triceps sural after a football game.

F. Consideration of muscle electro-stimulation

a. The Eastern countries at the end of the 60s used the EEM for the increase of the mus- cular mass. ${ }^{8}$

b. Its usefulness in the field of muscle rehabilitation and recovery, as well as complement to strength training in sports performance, is beyond doubt. ${ }^{9}$

c. EEM is effective in heavy legs, cramps and muscle contractures.

d. The EEM is right to exist if it is complemented with other training techniques.

\section{Materials and method}

Research was conducted on the effects of MSE applied to muscle injuries. Grade I, II, and minor injuries such as blows without acute stage ruptures of the lesion, the evolution was evaluated by clinical and imaging tests of said patients (Figures 1-4). It was carried out in patients, athletes, soccer players (Club Pachuca de Fútbol y National Team of Mexico), during the years 2004 and to date it is still applying. Electrostimulator brand Compex model 400.

3D Ultrasound Device (ultrasound) to verify the mobility of fibers against electrical stimuli, at different intensities of application and different programs. Acute and Regeneration phase. Low frequency stimulation. 1 to $10 \mathrm{~Hz}$ acute up to $50 \mathrm{~Hz}$ Regeneration. Stimulus time 300 to $400 \mathrm{~m} / \mathrm{s}$. Two-phase and rectangular pulse.

Intensity tolerance to the patient, from 20 to 30 MA very good stimulus, tested under excellent muscle ultrasound mobilization of fibers from 7 to $10 \mathrm{~cm}$. of depth. Important: Avoid maximum muscle tetanization that really is a Muscular Mobilization. 


\section{Treatment in different phases of muscle injury}

a. Acute phase ( 24 to $72 \mathrm{hs}$ ) from 1 to $10 \mathrm{~Hz}$.

b. Wanted effects: decreased pain, muscle relaxation, increased secretion of endorphins, increased blood flow, elimination of accumulated edema and metabolites, accelerated venous return combined with cryotherapy (massage). Regeneration phase. (up to high kinesics) This phase depends on each patient and degree of muscle injury. It can go from 5 to 21 days. From 10 to $50 \mathrm{~Hz}$.

c. Effects sought: Increase of electrical stimuli for better resistance to fatigue, mainly slow fibers (STI), increased muscle tone.

d. Combined with thermotherapy (Figure 5) maintained passive stretching (Figure 6).

e. High kinesic phase: Starts at the end of the previous one and lasts until medical discharge. (EEM combination plus Specific Entity in coordination with Physical Trainer) from 50 to $100 \mathrm{~Hz}$. Strengthening exercise with EEM (Figure 7) Bicycle with EEM (Figure 8).

f. Wanted Effects: Stimulation of fast fibers (type II) and interme diates, stimulates strength and localized resistance. Stimulation Time 20 mint.

g. Application up to 3 times per day.

h. Good placement of electrodes, stimulation without pain.

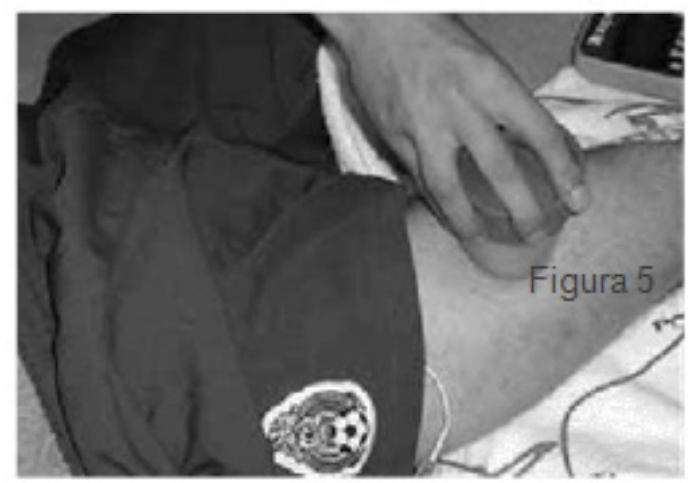

Figure 5

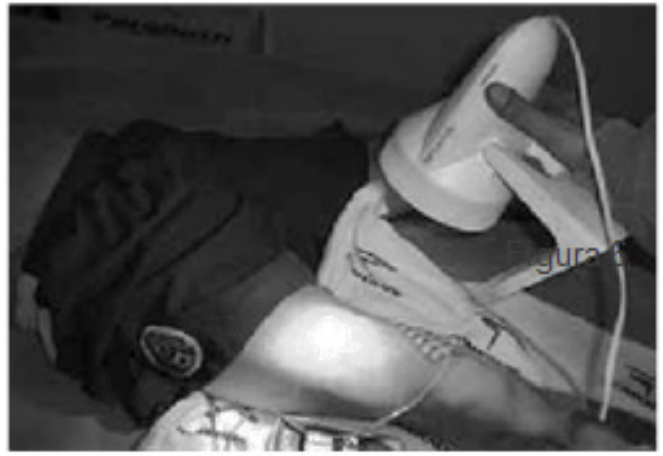

Figure 6

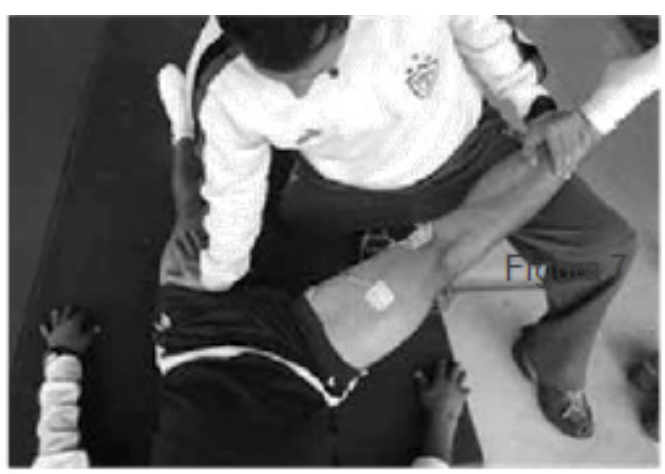

Figure 7

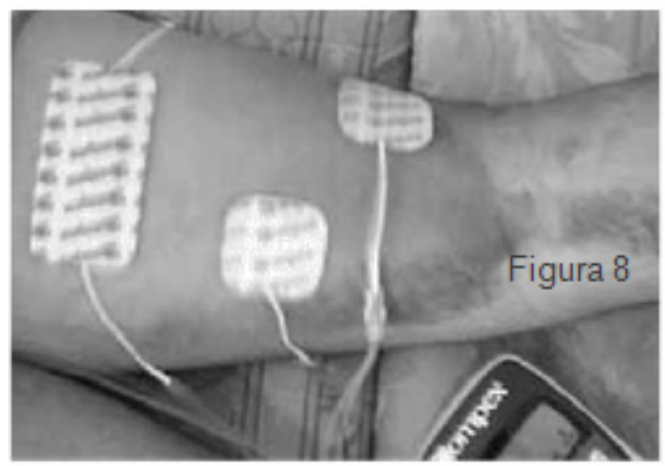

Figure 8

Suggested programs for treatment

\begin{tabular}{ll}
\hline Acute phase & Regenerative phase: \\
Endorphinic $6 \mathrm{~Hz}$ & Active recovery \\
Decontracting $2 \mathrm{~Hz}$ & Post-effort Restitution \\
Capillarization $8 \mathrm{~Hz}$ & Heavy legs \\
Sewer system & \\
\hline
\end{tabular}

i. Kinesics High Phase: Strength resistance $50-70 \mathrm{~Hz}, 300$ to 750 micro/s (Figure 9).

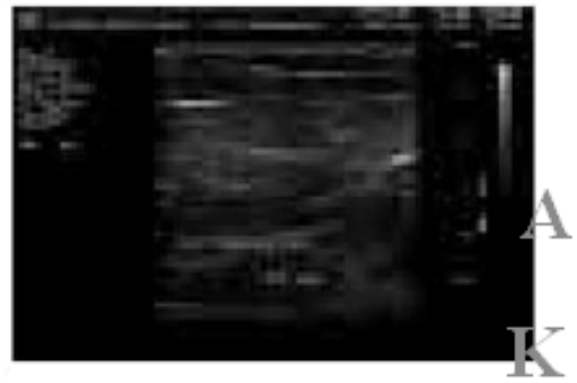

Figure 9

Under USG control in real time the response of the muscle fibers to the EEM was appreciated, in this image under the program (Endorphinic at $20 \mathrm{~m} \mathrm{I}$ ) they reacted by performing undulating rhythmic contractions, which covered the entire stimulated area reaching a depth of $10 \mathrm{~cm}$. cm., noticing a large increase in blood flow in the area to be treated. 
Comments: When I Increase the Freq. Hz., Decreases the stimulation time, this is different according to the body area to be treated, this already comes within the program to choose.

i. Contraindications If there is a muscle injury with a severity III damage, the treatment is contraindicated in the acute stage. Pac. Cardiac (thorax), Pregnant (abdomen).

ii. Major skin lesions (care where I apply the electrodes).

iii. Patients considered by the Physiotherapist not fit for the use of the EEM.

\section{Conclusion}

The treated patients were 53, with an average age of 25 years, they reported a great improvement, mainly in the early stages where muscle spasm decreased markedly and thus the symptom of pain that is why we emphasize the important use in the first stages of the muscular injury (acute).

1. The EEM guarantees a good quality of the regeneration of injured muscular tissue (adequate homeostasis) by stimulating the proliferative cells from the initial moment of the lesion, this condition improves the arrival of regenerative enzymes type: proteases, hydroases, lactacidoses.

2. Helps reduce post-injury pain, prevent muscle atrophy, edema and improve local circulation.

3. We maintain mediated electrical stimuli the normal physiological Biochemical processes.

a. Muscle directly converts chemical energy into dynamic energy. Myosin -Actin (help anchoring muscle fibers), Troponin -Tropomyosin (favor ion exchange to give the phenomenon of anchoring and disengagement).

b. Theory of sliding filaments, interaction of the proteins that constitute the meofibril under the stimulus that depolarizes these fibers.

c. Having the EEM consequences such as: Increase intracellular fluid by multiplying by 5 the number of capillaries. Increase in superficial exchange between blood and fibers.

d. Better diffusion of glucose and oxygen, better intracellular condition for regeneration.

e. Development of capillaries especially around muscle fibers. f. Better condition of Fatigue Resistance.

4. The EEM is active, the person feels, notices and endures the involuntary muscular contraction.

5. It was demonstrated under the ultrasound that the effectiveness of the EEM is in relation to the intensity applied to greater intensity greater activated motor neurons (30ma base to $120 \mathrm{ma}$ ).

6. The EEM helps a better condition of muscle regeneration.

7. Combined with active stretching increases muscle elasticity.

8. Combined with strength training and muscular strength improves the condition of it for an optimal return to activities.

9. The EEM is right to exist if it is complemented with other techniques of treatment.

The EEM is another tool in the treatment of muscle injuries.

Virtue and muscle are very similar, both need exercises to strengthen themselves. ${ }^{10-12}$

\section{Acknowledgment}

Thanks to Dr. Julio Cesar Martínez Morales of Central Radiologica Pachuca - Mexico, for his support in the realization of muscle ultrasounds and the editing video.

\section{References}

1. Salter R, 1985 .

2. Knigth, 1985.

3. Baker, 1988.

4. Lieber, 1988.

5. Tabar,1972.

6. Kenwright J, Simpson HA, 1991.

7. Jarvinen, 1975.

8. M Portman, 1976.

9. Crime, 1989.

10. L Ricchiuti, 2000

11. R Sassi, 1999.

12. A Lanzani, 2000. 